\title{
A Comparative Approach to the Solution of the Zabolotskaya-Khokhlov Equation by Iteration Methods
}

\author{
Saeed Ahmed and Muhammad Kalim \\ National College of Business Administration and Economics, Gulberg-III, 54660 Lahore, Pakistan \\ Correspondence should be addressed to Saeed Ahmed; saeedahmedshah54@gmail.com
}

Received 9 October 2015; Revised 26 December 2015; Accepted 27 December 2015

Academic Editor: Soheil Salahshour

Copyright ( 92016 S. Ahmed and M. Kalim. This is an open access article distributed under the Creative Commons Attribution License, which permits unrestricted use, distribution, and reproduction in any medium, provided the original work is properly cited.

We employed different iteration methods like Homotopy Analysis Method (HAM), Adomian Decomposition Method (ADM), and Variational Iteration Method (VIM) to find the approximate solution to the Zabolotskaya-Khokhlov (ZK) equation. Iteration methods are used to solve linear and nonlinear PDEs whose classical methods are either very complex or too limited to apply. A comparison study has been made to see which of these methods converges to the approximate solution rapidly. The result revealed that, amongst these methods, $\mathrm{ADM}$ is more effective and simpler tool in its nature which does not require any transformation or linearization.

\section{Introduction}

In science, physics, and engineering, many problems are modeled by the means of nonlinear evolutions equations especially in plasma physics, fluid dynamics, biology, and nonlinear acoustics. In this paper, we consider one such nonlinear partial differential equation which is $(1+1)$ dimensional Zabolotskaya-Khokhlov (ZK) equation (ZK for short)

$$
u_{x t}=u u_{x x}+\alpha\left(u_{x}\right)^{2} .
$$

This equation is one of the basic equations in nonlinear acoustic and nonlinear wave theory. It is named after two Russian mathematicians R. V. Khokhlov and E. A. Zabolotskaya who derived it for the first time almost four decades ago and presented an approximate solution which described some interesting features of nonlinear wave beams [1]. In recent years, an extensive research is being made in nonlinear acoustic due to the development of new medical devices for nonlinear diagnostic ultrasound imaging, for noninvasive destruction of tumors in acoustic surgery and for kidney stone comminution. All of the above applications rely on the focusing acoustic waves in nonlinear medium which can be studied by the ZK equation [2]. The ZK equation describes the propagation of a confined wave beam or sound beam in nonlinear medium without dispersion or absorption and it investigates the beam deformation associated with the properties of the nonlinear medium $[3,4]$. This equation has applications in many fields of life such as it is used to simulate the estimation of fish stock abundance, discrimination between fish species, the effect of excess attenuation which occurs due to the nonlinear sound propagation in water, absorption, and diffraction in the focused sound beams [58].

The solution of the ZK equation will shed light on some new features in the behavior of nonlinear beam. Many authors $[9,10]$ have found the infinitesimal symmetries and exact solution of the ZK equation by means of Lie Algebras, but the analytical or approximate solution of the ZK equation in the form of an infinite series has not been obtained before. Keeping this limitation in our mind, in this work, we will find the analytical solution of the ZK equation in the form of an infinite series with the help of iteration methods. This analytical solution would be extremely useful in the areas of theoretical physics, applied mathematics, and engineering as it will express the acoustic pressure of the beam in terms of propagation coordinate and time explicitly.

As we know that many physical problems are modeled by means of partial differential equations (PDEs) like Helmholtz equation, heat equation, wave equation, gas equation, and so forth [11-15]. Of these PDEs, some are linear, but many 
are quasi-linear or nonlinear [16] whose analytical solution techniques are limited to apply. There is no general method of solving such PDEs; however an extensive research is being made to find exact or approximate solutions of nonlinear partial differential equations with the aid of iteration methods like variational principal [17], decomposition solution [18, 19], and Adomian Decomposition Method [20-23]. These iteration methods are analytical tool that approximate the solution of such PDEs in terms of an infinite series which converges rapidly to the exact solution of the PDE in just few iterations. The accuracy and the speed of convergence of the resulting series are surprisingly high and one needs only a few iterations to approximate the exact solution as shown by the example given in Section 4.

Consider the initial and boundary conditions for (A)

$$
\begin{gathered}
u_{x}(x, 0)=f(x), \\
u(0, t)=g(t),
\end{gathered}
$$

where $f(x)$ and $g(t)$ are two known functions.

Integrating (A) twice and applying the initial conditions (1), we can write

$$
\begin{aligned}
u(x, t)= & F(x, t)+\int_{0}^{x} \int_{0}^{t} F_{1}(u(\zeta, \tau)) d \tau d \zeta \\
& +\alpha \int_{0}^{x} \int_{0}^{t} F_{2}(u(\zeta, \tau)) d \tau d \zeta
\end{aligned}
$$

where

$$
\begin{aligned}
F(x, t) & =g(t)+\int_{0}^{x} f(\zeta) d \zeta, \\
F_{1}(u(x, t)) & =u(x, t) \frac{\partial^{2} u(x, t)}{\partial x^{2}}, \\
F_{2}(u(x, t)) & =\left(\frac{\partial u(x, t)}{\partial x}\right)^{2} .
\end{aligned}
$$

In (2), we assume that $F(x, t)$ is bounded function for all $t$ in $[0, T]$ and $x$ in $[a, b]$, where $T, a, b \in \mathbb{R}$. The functions $F_{1}(u(x, t))$ and $F_{2}(u(x, t))$ are Lipschitz continuous such that

$$
\begin{aligned}
\left|F_{i}(u)-F_{i}\left(u^{*}\right)\right| \leq K_{i}\left|u-u^{*}\right|, \quad i=1,2, & \\
k_{1} & =T(b-a)\left(\alpha K_{1}+K_{2}\right), \\
k_{2} & =1-T\left(1-k_{1}\right) .
\end{aligned}
$$

\section{Existence and Uniqueness}

In this section, we will analyze uniqueness and existence of the solution to the $\mathrm{ZK}$ equation.

Theorem 1. Equation (A) has a unique solution for $0<k_{1}<1$.
Proof. We suppose that $u$ and $u^{*}$ are two solutions of (2), and then

$$
\begin{aligned}
& \left|u-u^{*}\right|=\mid\left(F(x, t)+\int_{0}^{x} \int_{0}^{t} F_{1}(u(\zeta, \tau)) d \tau d \zeta\right. \\
& \left.+\alpha \int_{0}^{x} \int_{0}^{t} F_{2}(u(\zeta, \tau)) d \tau d \zeta\right)-(F(x, t) \\
& +\int_{0}^{x} \int_{0}^{t} F_{1}\left(u^{*}(\zeta, \tau)\right) d \tau d \zeta \\
& \left.+\alpha \int_{0}^{x} \int_{0}^{t} F_{2}\left(u^{*}(\zeta, \tau)\right) d \tau d \zeta\right) \\
& \left|u-u^{*}\right| \leq \int_{0}^{x} \int_{0}^{t}\left|F_{1}(u(\zeta, \tau))\right| d \tau d \zeta \\
& +\alpha \int_{0}^{x} \int_{0}^{t}\left|F_{2}(u(\zeta, \tau))\right| d \tau d \zeta \\
& -\int_{0}^{x} \int_{0}^{t}\left|F_{1}\left(u^{*}(\zeta, \tau)\right)\right| d \tau d \zeta \\
& -\alpha \int_{0}^{x} \int_{0}^{t}\left|F_{2}\left(u^{*}(\zeta, \tau)\right)\right| d \tau d \zeta \\
& \left|u-u^{*}\right| \leq \int_{0}^{x} \int_{0}^{t}\left|F_{1}(u(\zeta, \tau))-F_{1}\left(u^{*}(\zeta, \tau)\right)\right| d \tau d \zeta \\
& +\alpha \int_{0}^{x} \int_{0}^{t}\left|F_{2}(u(\zeta, \tau))-F_{2}\left(u^{*}(\zeta, \tau)\right)\right| d \tau d \zeta, \\
& \left|u-u^{*}\right| \leq\left(K_{1}+K_{2} \alpha\right) T(b-a)\left|u(\zeta, \tau)-u^{*}(\zeta, \tau)\right|, \\
& \left|u-u^{*}\right| \leq k_{1}\left|u-u^{*}\right|, \\
& \left(1-k_{1}\right)\left|u-u^{*}\right| \leq 0 .
\end{aligned}
$$

Since $0<k_{1}<1,\left|u-u^{*}\right|=0 \Rightarrow u=u^{*}$ which proves that (A) has a unique solution.

\section{Analysis of the Methods}

In this section, we will introduce the iteration methods which we will apply on the ZK equation. We will apply Homotopy Analysis Method (HAM), Adomian Decomposition Method (ADM), and Variational Iteration Method (VIM) to solve the said equation.

3.1. Homotopy Analysis Method (HAM). To illustrate the basic idea of the Homotopy Analysis Method, we consider the following nonlinear differential equation:

$$
A(u(x, t))=0,
$$

where $A$ is any differential or integral operator and $u(x, t)$ is the unknown function. Using Homotopy Analysis Method, we can construct the so-called zeroth-order deformation equation

$$
\begin{gathered}
(1-p)\left[L(\phi(x, t, p))-L\left(u_{0}(x, t)\right)\right] \\
=p h H(x, t) N(\phi(x, t, p)),
\end{gathered}
$$


where $u_{0}(x, t)$ is an initial guess to the exact solution $u(x, t)$ of the given differential equation and $p \in[0,1]$ is the embedding parameter, whereas $h \neq 0$ is an auxiliary parameter, $L$ is an auxiliary linear operator, $H(x, t)$ is an auxiliary function, and $\phi(x, t, p)$ is the unknown function. Observe that when $p=0$, relation (7) turns out to be $\phi(x, t, 0)=u_{0}(x, t)$, and when $p=1$ it becomes $\phi(x, t, 1)=$ $u(x, t)$. So, as the embedding parameter $p$ changes from 0 to 1 , we see that $\phi(x, t, p)$ continuously changes from the initial condition $u_{0}(x, t)$ to the exact solution $u(x, t)$ of the differential equations.

Using Taylor theorem, we can express $\phi(x, t, p)$ as a power series of $p$

$$
\phi(x, t, p)=u_{0}(x, t)+\sum_{k=1}^{\infty} p^{k} u_{k}
$$

where

$$
u_{k}(x, t)=\left.\frac{1}{k !} \frac{\partial^{k} \phi(x, t, p)}{\partial x^{k}}\right|_{p=0} .
$$

It is obvious that the convergence of the above relation depends mainly on the auxiliary parameter $h$. With the proper choice of the initial guess $u_{0}(x, t)$, the power series (8) converges to the exact solution at $p=1$

$$
u(x, t)=\phi(x, t, 1)=u_{0}(x, t)+\sum_{k=1}^{\infty} u_{k} .
$$

Next, we substitute (8) into (7) to get

$$
(1-p) L\left[\sum_{k=1}^{\infty} p^{k} u_{k}\right]=p h H(x, t) N(\phi(x, t, p)) .
$$

Differentiating (11) $k$ times with respect to $p$ and dividing both sides by $k$ ! and finally applying $p=0$, we can write

$$
\begin{aligned}
& \frac{\partial^{k}}{\partial p^{k}}\left((1-p) L\left[\sum_{k=1}^{\infty} p^{k} u_{k}\right]\right) \\
& \quad=\frac{\partial^{k}}{\partial p^{k}}[p h H(x, t) N(\phi(x, t, p))], \\
& \frac{\partial^{k}}{\partial p^{k}}\left(L\left[\sum_{k=1}^{\infty} p^{k} u_{k}\right]\right)-\frac{\partial^{k}}{\partial p^{k}}\left(p L\left[\sum_{k=1}^{\infty} p^{k} u_{k}\right]\right) \\
& \quad=\frac{\partial^{k}}{\partial p^{k}}[p h H(x, t) N(\phi(x, t, p))], \\
& L\left[u_{k}(x, t)-\chi_{k} u_{k-1}(x, t)\right] \\
& \quad=h H(x, t) R_{k}\left[u_{k-1}(x, t)\right],
\end{aligned}
$$

where

$$
\begin{aligned}
\chi_{k} & = \begin{cases}0, & k \leq 1 \\
1, & k>1,\end{cases} \\
R_{k}\left[u_{k-1}(x, t)\right] & =\left.\frac{1}{(k-1) !} \frac{\partial^{k-1} N[\phi(x, t, p)]}{\partial x^{k-1}}\right|_{p=0} .
\end{aligned}
$$

The higher order deformation equation (12) governs $L$ and so $R_{k}\left[u_{k-1}(x, t)\right]$ is valid for any nonlinear operator $N$. So, we can let

$$
\begin{aligned}
N[\phi(x, t)]= & u(x, t)-F(x, t) \\
& -\int_{0}^{x} \int_{0}^{t} F_{1}\left(u_{k}(\zeta, \tau)\right) d \tau d \zeta \\
& -\alpha \int_{0}^{x} \int_{0}^{t} F_{2}\left(u_{k}(\zeta, \tau)\right) d \tau d \zeta
\end{aligned}
$$

which then becomes

$$
\begin{aligned}
R_{k}\left[u_{k-1}(x, t)\right]= & u_{k-1}(x, t)-F(x, t) \\
& -\int_{0}^{x} \int_{0}^{t} F_{1}\left(u_{k}(\zeta, \tau)\right) d \tau d \zeta \\
& -\alpha \int_{0}^{x} \int_{0}^{t} F_{2}\left(u_{k}(\zeta, \tau)\right) d \tau d \zeta .
\end{aligned}
$$

Substituting (15) into (12), we have

$$
\begin{gathered}
L\left[u_{k}(x, t)-\chi_{k} u_{k-1}(x, t)\right]=h H(x, t)\left(u_{k-1}(x, t)\right. \\
-F(x, t)-\int_{0}^{x} \int_{0}^{t} F_{1}\left(u_{k}(\zeta, \tau)\right) d \tau d \zeta \\
\left.-\alpha \int_{0}^{x} \int_{0}^{t} F_{2}\left(u_{k}(\zeta, \tau)\right) d \tau d \zeta\right) .
\end{gathered}
$$

Now, taking $F(x, t)$ as initial guess $u_{0}(x, t), h=-1, \alpha=1$, and $H(x, t)=1$, together with using the value of $\chi_{k}$, we can write the recurrence relation from the last equation as follows:

$$
\begin{aligned}
u_{0}(x, t)= & F(x, t) \\
u_{k+1}(x, t)= & \int_{0}^{x} \int_{0}^{t} F_{1}\left(u_{k}(\zeta, \tau)\right) d \tau d \zeta \\
& +\int_{0}^{x} \int_{0}^{t} F_{2}\left(u_{k}(\zeta, \tau)\right) d \tau d \zeta \quad k \geq 1 .
\end{aligned}
$$

The set of equations in (17) can be solved by simple integration. The resulting components $u_{i}, i=1,2, \ldots$ will then be substituted in (8) with $p$ as unity, which will lead towards the solution of (A) in the form of an infinite series whose convergence is sure in most cases in its own domain.

As we see, the initial and boundary conditions play vital role in determining the series solution and different set of conditions will result in different series solutions. Though we do not have general criteria of imposing restrictions at these conditions at the start, one should be careful in choosing initial conditions.

3.2. Adomian Decomposition Method (ADM). Adomian Decomposition Method was first introduced by Adomian in $1984[18,19]$. Like HAM, in this method, we split the nonlinear partial differential equation into two parts, namely, linear and nonlinear. Applying the inverse linear operator on both sides 
of the equation and decomposing the unknown function in the form of a series, we get a solution. In ADM, we also decompose the nonlinear terms in the form of Adomian polynomials and use a special formula to find these unknown terms due to which, no doubt, we need to do extra calculation than we do in HAM.

Consider the following equation:

$$
A(u(x, t))=0 .
$$

Equation (18) can be split into the following form:

$$
L(u(x, t))+N(u(x, t))=0,
$$

where $L$ and $N$ are linear and nonlinear differential operators, respectively. Consider that the inverse operator $L^{-1}$ exists, and applying it on (19), we get

$$
u(x, t)=u_{0}(x, t)-L^{-1}(N[u(x, t)]),
$$

where $u_{0}(x, t)$ is the initial guess of the equation.

By the Adomian Decomposition Method [16], we can define the unknown function

$$
u=\sum_{k=0}^{\infty} u_{k}
$$

The nonlinear term of the equation is given by the formula [18]

$$
N=\sum_{n=0}^{\infty} A_{n}, \quad n \geq 0,
$$

where $A_{n}, n \geq 0$ are the Adomian polynomials which can be retrieved by the formula

$$
A_{n}\left(u_{0}, u_{1}, \ldots, u_{n}\right)=\frac{1}{n !} \frac{d^{n}}{d \lambda^{n}}\left[N\left(\sum_{i=0}^{n} \lambda^{i} u_{i}\right)\right]_{\lambda=0} .
$$

Substituting (21) and (22) into (20), we can write

$$
\sum_{k=0}^{\infty} u_{k}(x, t)=u_{0}(x, t)-L^{-1}\left(\sum_{n=0}^{\infty} A_{n}\right) .
$$

Relation (24) can be used to determine the solution of (18).

3.3. Variational Iteration Method (VIM). Under Variational Iteration Method, we break the given (6) into the following form:

$$
N(u(x, t))+L(u(x, t))=0
$$

where $N$ and $L$ are nonlinear and linear operators, respectively. According to VIM, a correctional functional can be constructed as follows:

$$
\begin{aligned}
u_{n+1} & (x, t) \\
= & u_{n}(x, t) \\
& +\int_{0}^{t} \lambda(x, \tau)\left[N\left(u_{n}(x, \tau)\right)+L\left(u_{n}(x, \tau)\right)\right] d \tau,
\end{aligned}
$$

where $\lambda$ is the Lagrange multiplier which can be obtained optimally by using variation theory. The function $u_{n}(x, t)$ is the restricted variation; that is $\delta u_{n}(x, t)=0$, which helps us to determine the Lagrange multiplier together with integration by parts. The exact solution may be obtained by

$$
u(x, t)=\lim _{n \rightarrow \infty} u_{n}(x, t) .
$$

Applying (26) on (2), we can write

$$
\begin{aligned}
& u_{n+1}(x, t)=u_{n}(x, t)+L^{-1}\left[\lambda \left\{u_{n}(x, t)-F(x, t)\right.\right. \\
& -\int_{0}^{x} \int_{0}^{t} F_{1}\left(u_{n}(\zeta, \tau)\right) d \tau d \zeta \\
& \left.\left.-\alpha \int_{0}^{x} \int_{0}^{t} F_{2}\left(u_{n}(\zeta, \tau)\right) d \tau d \zeta\right\}\right], \quad n \geq 0,
\end{aligned}
$$

where $L^{-1}[\cdot]=\int_{0}^{t}[\cdot] d \tau$.

Next we find the value of Lagrange multiplier by taking variation on (28)

$$
\begin{aligned}
& \delta u_{n+1}(x, t)=\delta u_{n}(x, t)+\delta L^{-1}\left[\lambda \left\{u_{n}(x, t)-F(x, t)\right.\right. \\
& -\int_{0}^{x} \int_{0}^{t} F_{1}\left(u_{n}(\zeta, \tau)\right) d \tau d \zeta \\
& \left.\left.-\alpha \int_{0}^{x} \int_{0}^{t} F_{2}\left(u_{n}(\zeta, \tau)\right) d \tau d \zeta\right\}\right], \quad n \geq 0 .
\end{aligned}
$$

From (29), due to stationary conditions, we get, after a bit of calculations,

$$
\begin{array}{rlr}
\lambda^{\prime} & =0 \text { or } \\
1+\lambda & =0
\end{array}
$$

which means that the Lagrange multiplier is -1 , so from (28) we have

$$
\begin{aligned}
& u_{n+1}(x, t)=u_{n}(x, t)-L^{-1}\left[u_{n}(x, t)-F(x, t)\right. \\
& -\int_{0}^{x} \int_{0}^{t} F_{1}\left(u_{n}(\zeta, \tau)\right) d \tau d \zeta \\
& \left.-\alpha \int_{0}^{x} \int_{0}^{t} F_{2}\left(u_{n}(\zeta, \tau)\right) d \tau d \zeta\right], \quad n \geq 0 .
\end{aligned}
$$

We can find the successive values of $u_{n}(x, t)$ from relation (31).

3.4. Theorem. The series solution $u(x, t)=\sum_{k=0}^{\infty} u_{k}(x, t)$ using VIM converges with $0<k_{1}<1,0<k_{2}<1$.

Proof. Consider

$$
\begin{aligned}
& u_{n+1}(x, t)=u_{n}(x, t)-L^{-1}\left[u_{n}(x, t)-F(x, t)\right. \\
& -\int_{0}^{x} \int_{0}^{t} F_{1}\left(u_{n}(\zeta, \tau)\right) d \tau d \zeta \\
& \left.-\alpha \int_{0}^{x} \int_{0}^{t} F_{2}\left(u_{n}(\zeta, \tau)\right) d \tau d \zeta\right] .
\end{aligned}
$$


Removing the subscripts, we can again write

$$
\begin{gathered}
u(x, t)=u(x, t)-L^{-1}[u(x, t)-F(x, t) \\
-\int_{0}^{x} \int_{0}^{t} F_{1}(u(\zeta, \tau)) d \tau d \zeta \\
\left.-\alpha \int_{0}^{x} \int_{0}^{t} F_{2}(u(\zeta, \tau)) d \tau d \zeta\right]
\end{gathered}
$$

Subtracting (33) from (32), we get

$$
\begin{aligned}
& u_{n+1}(x, t)-u(x, t)=u_{n}(x, t)-u(x, t) \\
& -L^{-1}\left[u_{n}(x, t)-u(x, t)\right. \\
& -\int_{0}^{x} \int_{0}^{t}\left[F_{1}\left(u_{n}(\zeta, \tau)\right)-F_{1}(u(\zeta, \tau))\right] d \tau d \zeta \\
& \left.-\alpha \int_{0}^{x} \int_{0}^{t}\left[F_{2}\left(u_{n}(\zeta, \tau)\right)-F_{2}(u(\zeta, \tau))\right] d \tau d \zeta\right] .
\end{aligned}
$$

Now, let

$$
\begin{aligned}
e_{n+1} & =u_{n+1}(x, t)-u(x, t), \\
e_{n} & =u_{n}(x, t)-u(x, t), \\
\left|e_{n}\left(x, t^{*}\right)\right| & =\max _{t} e_{n}(x, t) .
\end{aligned}
$$

Now, observe that $e_{n}$ is decreasing with respect to $t$, so applying mean value theorem, we can write from (34) the following:

$$
\begin{aligned}
& e_{n+1}(x, t)=e_{n}(x, t)-L^{-1}\left[e_{n}(x, t)\right. \\
& -\int_{0}^{x} \int_{0}^{t}\left[F_{1}\left(u_{n}(\zeta, \tau)\right)-F_{1}(u(\zeta, \tau))\right] d \tau d \zeta \\
& \left.-\alpha \int_{0}^{x} \int_{0}^{t}\left[F_{2}\left(u_{n}(\zeta, \tau)\right)-F_{2}(u(\zeta, \tau))\right] d \tau d \zeta\right], \\
& e_{n+1}(x, t) \leq e_{n}(x, t)-L^{-1}\left[e_{n}(x, t)\right. \\
& \left.\quad-L^{-1}\left|e_{n+1}(x, t)\right| T(b-a)\left(\alpha K_{1}-K_{2}\right)\right], \\
& e_{n+1}(x, t) \leq e_{n}(x, t)-\left[T e_{n}(x, \eta)\right. \\
& \left.-T(b-a)\left(\alpha K_{1}-K_{2}\right) L^{-1} L^{-1}\left|e_{n+1}(x, t)\right|\right], \\
& e_{n+1}(x, t) \leq e_{n}(x, t)-T\left(1-k_{1}\right)\left|e_{n+1}\left(x, t^{*}\right)\right|, \\
& e_{n+1}(x, t) \leq\left(1-T\left(1-k_{1}\right)\right)\left|e_{n+1}\left(x, t^{*}\right)\right|, \\
& e_{n+1}(x, t) \leq k_{2}\left|e_{n+1}\left(x, t^{*}\right)\right| .
\end{aligned}
$$

Therefore,

$$
\begin{aligned}
\left\|e_{n+1}(x, t)\right\| & =\max \left|e_{n+1}(x, t)\right| \leq k_{2} \max \left\|e_{n}\left(x, t^{*}\right)\right\| \\
& \leq k_{2}\left\|e_{n}\left(x, t^{*}\right)\right\| .
\end{aligned}
$$

Since $0<k_{2}<1,\left\|e_{n}\left(x, t^{*}\right)\right\| \rightarrow 0$, so the series converges and hence the proof.

\section{Application and Results}

In this section, we solve the $\mathrm{ZK}$ equation by the abovementioned methods. We also compare the results obtained to see which method is more sufficient and converges rapidly to the exact solution.

4.1. Example. Consider ZK equation (A) with $\alpha=1$ together with initial and boundary conditions given as follows:

$$
\begin{gathered}
u_{x t}=u u_{x x}+\left(u_{x}\right)^{2}, \\
f(x)=2 x, \quad g(t)=0 .
\end{gathered}
$$

Here $u=u(x, t)$ is the unknown function to be determined which represents the acoustic pressure and $x$ is the propagation coordinate. The term $u_{x t}$ denotes the time evolution of the wave, while the nonlinear terms $u u_{x x}$ and $u_{x}^{2}$ describe the steepening of the wave.

Using HAM. We apply HAM on (38) to get the following iteration scheme due to (17):

$$
\begin{aligned}
u_{0}(x, t)= & F(x, t)=g(t)+\int_{0}^{x} f(\zeta) d \zeta \\
= & 0+\int_{0}^{x} 2 \zeta d \zeta=x^{2} \\
u_{k+1}(x, t)= & +\int_{0}^{x} \int_{0}^{t} F_{1}\left(u_{k}(\zeta, \tau)\right) d \tau d \zeta \\
& +\int_{0}^{x} \int_{0}^{t} F_{2}\left(u_{k}(\zeta, \tau)\right) d \tau d \zeta \quad k \geq 1 .
\end{aligned}
$$

Using the above relation, we can find the other components as follows:

$$
\begin{aligned}
& u_{1}(x, t)=2 x^{3} t, \\
& u_{2}(x, t)=4 x^{4} t^{2}, \\
& u_{3}(x, t)=\frac{80}{7} x^{9} t^{7}, \\
& u_{4}(x, t)=\frac{3840}{49} x^{17} t^{15} .
\end{aligned}
$$

Substituting these values in (8) and making $p$ unity, we get the solution of (38) in the form of the following infinite series:

$$
\begin{aligned}
u(x, t)= & \sum_{n=0}^{\infty} u_{n} \\
= & x^{2}+2 x^{3} t+4 x^{4} t^{2}+\frac{80}{7} x^{9} t^{7} \\
& +\frac{3840}{49} x^{17} t^{15} \cdots .
\end{aligned}
$$


This represents the acoustic pressure of the beam in terms of propagation coordinate and time.

Using $A D M$. Let $L=\partial^{2} / \partial x \partial t$ and $N=u u_{x x}+\left(u_{x}\right)^{2}$ so that (38) can be written as

$$
L[u]=N[u]
$$

Consider that the inverse operator $L^{-1}$ exists and is defined as

$$
L^{-1}[\cdot]=\int_{0}^{x} \int_{0}^{t}[\cdot] d t d x
$$

Applying $L^{-1}$ on (42) and using the initial conditions, we get

$$
u(x, t)=x^{2}+L^{-1}(N[u]) .
$$

By the Adomian Decomposition Method, we can define the unknown function

$$
u=\sum_{k=0}^{\infty} u_{k}
$$

The nonlinear term of the equation is given by the formula

$$
N=\sum_{n=0}^{\infty} A_{n}, \quad n \geq 0
$$

where $A_{n}, n \geq 0$ are the Adomian polynomials which can be retrieved by the formula

$$
A_{n}\left(u_{0}, u_{1}, \ldots, u_{n}\right)=\frac{1}{n !} \frac{d^{n}}{d \lambda^{n}}\left[N\left(\sum_{i=0}^{n} \lambda^{i} u_{i}\right)\right]_{\lambda=0}
$$

Substituting (45) and (46) into (44), we can write

$$
\sum_{k=0}^{\infty} u_{k}(x, t)=x^{2}+L^{-1}\left(\sum_{n=0}^{\infty} A_{n}\right) .
$$

From (48), we get the recurrence relation as

$$
\begin{aligned}
u_{0}(x, t) & =x^{2}, \\
u_{n+1}(x, t) & =L^{-1}\left(A_{n}\right) .
\end{aligned}
$$

Now, using formula (47), we can find the first few Adomian polynomials as

$$
\begin{aligned}
& A_{0}=6 x^{2}, \\
& A_{1}=40 x^{3} t, \\
& A_{2}=210 x^{4} t^{2}, \\
& A_{3}=1008 x^{5} t^{3} .
\end{aligned}
$$

Applying the recurrence relation (49) together with the inverse operator (43) on the set of equations given in (50) to get the components of $u(x, t)$ and inserting these components in (45), we get the solution to (38) as

$$
\begin{gathered}
u_{0}=x^{2}, \\
u_{1}(x, t)=2 x^{3} t, \\
u_{2}(x, t)=5 x^{4} t^{2}, \\
u_{3}(x, t)=14 x^{5} t^{3}, \\
u_{4}(x, t)=42 x^{6} t^{4} .
\end{gathered}
$$

Substituting these values in (8) and making $p$ unity, we get the series

$$
\begin{aligned}
u(x, t) & =\sum_{n=0}^{\infty} u_{n} \\
& =x^{2}+2 x^{3} t+5 x^{4} t^{2}+14 x^{5} t^{3}+42 x^{6} t^{4}+\cdots
\end{aligned}
$$

The exact solution to (38) can be written in a closed form as follows:

$$
u(x, t)=\sum_{n=1}^{\infty} \frac{(2 n) !}{n !(n+1) !} x^{n+1} t^{n-1}=\frac{4 x^{2}}{(1+\sqrt{1-4 x t})^{2}}
$$

Using VIM. Using VIM, we can find the recurrence relation given as follows:

$$
\begin{aligned}
& u_{n+1}(x, t)=u_{n}(x, t)-L^{-1}\left[u_{n}(x, t)-F(x, t)\right. \\
& -\int_{0}^{x} \int_{0}^{t} F_{1}\left(u_{n}(\zeta, \tau)\right) d \tau d \zeta \\
& \left.-\alpha \int_{0}^{x} \int_{0}^{t} F_{2}\left(u_{n}(\zeta, \tau)\right) d \tau d \zeta\right], \quad n \geq 0
\end{aligned}
$$

Since

$$
\begin{aligned}
u_{0}(x, t) & =F(x, t)=g(t)+\int_{0}^{x} f(\zeta) d \zeta \\
& =0+\int_{0}^{x} 2 \zeta d \zeta=x^{2}
\end{aligned}
$$

we can do few more iterations and have the successive components of $u_{n+1}(x, t)$ as follows:

$$
\begin{aligned}
u_{1}(x, t)= & x^{2}+x^{3} t^{2} \\
u_{2}(x, t)= & x^{2}+\left(2 t^{2}-\frac{1}{3} t^{3}\right) x^{3}+\frac{5}{12} x^{4} t^{4}+\frac{1}{10} x^{5} t^{6} \\
u_{3}(x, t)= & x^{2}+\left(3 t^{2}-t^{3}+\frac{1}{12} t^{4}\right) x^{3} \\
& +\left(\frac{5}{4} t^{4}-\frac{1}{6} t^{5}\right) x^{4}
\end{aligned}
$$




$$
\begin{aligned}
& +\left(\frac{7}{12} t^{6}-\frac{23}{210} t^{7}+\frac{1}{168} t^{8}\right) x^{5} \\
& -\frac{35}{2592} x^{6} t^{9} \\
& +\left(\frac{413}{16200} t^{10}-\frac{2}{825} t^{11}\right) x^{7} \\
& +\left(\frac{7}{60} t^{6}+\frac{2}{352} t^{12}\right) x^{8}+\frac{1}{3640} x^{9} t^{14}
\end{aligned}
$$

Consequently, the third-order approximate solution of (38) using VIM is given by

$$
\begin{aligned}
u(x, t) \simeq & u_{3}(x, t) \\
= & x^{2}+\left(3 t^{2}-t^{3}+\frac{1}{12} t^{4}\right) x^{3} \\
& +\left(\frac{5}{4} t^{4}-\frac{1}{6} t^{5}\right) x^{4} \\
& +\left(\frac{7}{12} t^{6}-\frac{23}{210} t^{7}+\frac{1}{168} t^{8}\right) x^{5}-\frac{35}{2592} x^{6} t^{9} \\
& +\left(\frac{413}{16200} t^{10}-\frac{2}{825} t^{11}\right) x^{7} \\
& +\left(\frac{7}{60} t^{6}+\frac{2}{352} t^{12}\right) x^{8}+\frac{1}{3640} x^{9} t^{14}
\end{aligned}
$$

Table 1 shows the comparison between the approximate solutions (41), (51), and (57) and the exact solution (53) of (38). From the table, we see that the approximate solution obtained by $\mathrm{ADM}$ is closer to the exact solution than others. After ADM, the approximate solution by HAM is better than that of VIM. Since we take the third-order solution from VIM, it is suggested that higher order solution obtained by VIM may rapidly lead to convergence towards the solution. If we look at the table carefully, we will see that these approximate solutions are close to the exact one for the values $x=0.1$ to 0.3 and after this the approximate solutions seem to be moving away to the exact solution. Since the exact solution of the equation given in (53) has the domain $1-4 x t>$ $0 \Rightarrow x t<0.25$ and by fixing $t=0.2$, we get that the restriction on $x$ is $x<1.25$. So, as the values of $x$ are getting close to 1.25, the solution is diverging away.

Table 2 shows the absolute error of these solutions at different values of $x$. Again, we see that the approximate solution of ADM has less percentage error than others. The percentage error is increasing as $x$ is getting close to 1.25 due to the domain of $x$.

In Figure 1, the graph shows the approximate solutions by the iterations methods together with the exact solution (purple line). We see that the approximate solution by ADM (red line) is closer to the exact solution followed by HAM
TABLE 1: The values of $u(x, t)$ at $t=0.2, x=0.1-0.9$.

\begin{tabular}{lcccc}
\hline & $u_{\mathrm{HAM}}$ & $u_{\mathrm{ADM}}$ & $u_{\mathrm{VIM}}$ & Exact \\
\hline$x=0.1$ & 0.01040032 & 0.01042119 & 0.01011233 & 0.01042119 \\
$x=0.2$ & 0.04321024 & 0.04356014 & 0.04090019 & 0.04356076 \\
$x=0.3$ & 0.10087776 & 0.10274115 & 0.09304346 & 0.10275264 \\
$x=0.4$ & 0.18592772 & 0.19214213 & 0.16722674 & 0.19223594 \\
$x=0.5$ & 0.30100029 & 0.31705000 & 0.26413946 & 0.31754163 \\
$x=0.6$ & 0.44888979 & 0.4841644 & 0.3844759 & 0.48612181 \\
$x=0.7$ & 0.63258414 & 0.70194985 & 0.5289352 & 0.70843802 \\
$x=0.8$ & 0.85530539 & 0.98103624 & 0.69822148 & 1.00000000 \\
$x=0.9$ & 1.12055235 & 1.33466772 & 0.89304379 & 1.38562172 \\
\hline
\end{tabular}

TABLE 2: The absolute error of $u(x, t)$ at $t=0.2, x=0.1-0.9$.

\begin{tabular}{lccc}
\hline & $u_{\mathrm{HAM}}$ & $u_{\mathrm{ADM}}$ & $u_{\mathrm{VIM}}$ \\
\hline$x=0.1$ & 0.00002087171 & 0.00000000451 & 0.00030886334 \\
$x=0.2$ & 0.00035052253 & 0.00000062180 & 0.00266056974 \\
$x=0.3$ & 0.00187487820 & 0.00001149230 & 0.00029081445 \\
$x=0.4$ & 0.00187480000 & 0.00001150000 & 0.00970910000 \\
$x=0.5$ & 0.00630820000 & 0.00009380000 & 0.02500920000 \\
$x=0.6$ & 0.01654130000 & 0.00049160000 & 0.05340210000 \\
$x=0.7$ & 0.03723200000 & 0.00195740000 & 0.10164590000 \\
$x=0.8$ & 0.07585390000 & 0.00648810000 & 0.17950280000 \\
$x=0.9$ & 0.14469460000 & 0.01896380000 & 0.30177850000 \\
\hline
\end{tabular}

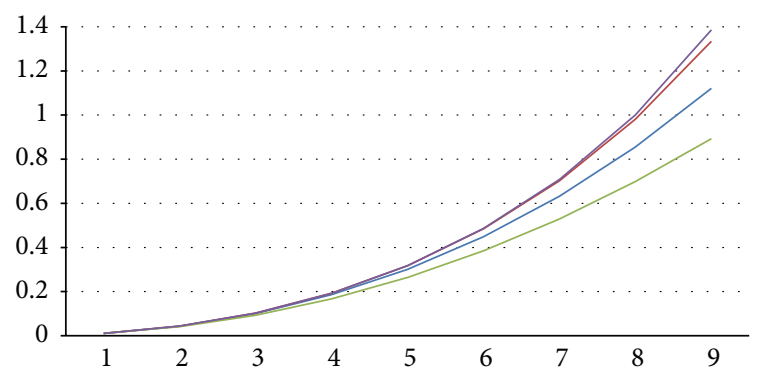

FIgure 1: The exact solution is in purple line, HAM is in blue, ADM is in red, and VIM is in green.

(blue line) and VIM (green line). In Figure 2, the error plot is given and we again see that the absolute error by ADM (red line) is the smallest, while the absolute error of VIM (green line) is the highest.

\section{Conclusion}

A comparative study has been made to seek the semianalytical solution of the ZK equation with initial and boundary conditions. The solution of the ZK equation is obtained in the form of an infinite series that converges rapidly in its domain. This form of solution gives the acoustic pressure of the beam in terms of propagation coordinate and time more explicitly. Since such form of the solution has not been obtained before, we believe that this will open new ways to understand the propagation of the confined beam in the nonlinear medium. Different iteration methods like HAM, ADM, and VIM have 


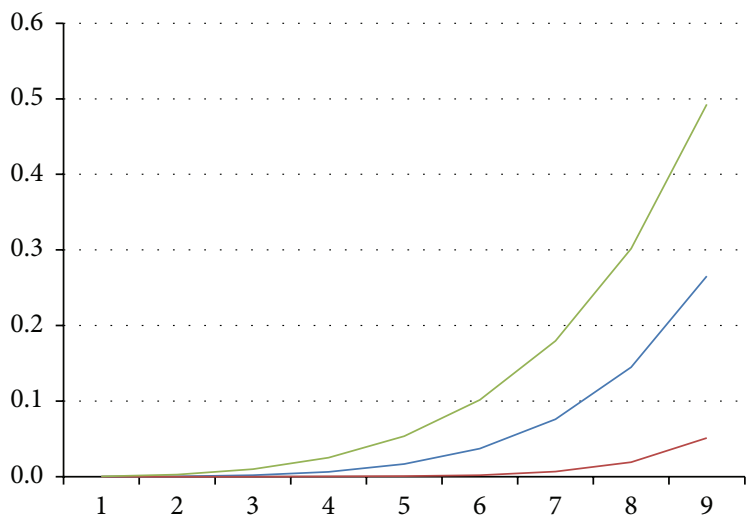

FIgURE 2: The error graph of HAM is in blue, ADM is in red, and VIM is in green.

been applied and compared. The result revealed that ADM generated surprisingly effective results.

\section{Conflict of Interests}

The authors declare that they there is no conflict of interests regarding the publication of this paper.

\section{References}

[1] O. V. Rudenko, "The 40th anniversary of the KhokhlovZabolotskaya equation," Acoustical Physics, vol. 56, no. 4, pp. 457-466, 2010.

[2] O. V. Bessonova, V. A. Khokhlova, M. R. Bailey, M. S. Canney, and L. A. Crum, "Focusing of high power ultrasound beams and limiting values of shock wave parameters," Acoustical Physics, vol. 55, no. 4-5, pp. 463-473, 2009.

[3] M. Kumar, R. Kumar, and A. Kumar, "On similarity solutions of Zabolotskaya-Khokhlov equation," Computers \& Mathematics with Applications, vol. 68, no. 4, pp. 454-463, 2014.

[4] M. Tajiri, "Similarity reductions of the Zabolotskaya-Khokhlov equation with a dissipative term," Journal of Nonlinear Mathematical Physics, vol. 2, no. 3-4, pp. 392-397, 1995.

[5] I. Kostin and G. Panasenko, "Khokhlov-ZabolotskayaKuznetsov-type equation: nonlinear acoustics in heterogeneous media," SIAM Journal on Mathematical Analysis, vol. 40, no. 2, pp. 699-715, 2008.

[6] A. Rozanova-Pierrat, "Qualitative analysis of the KhokhlovZabolotskaya-KUZnetsov (KZK) equation," Mathematical Models \& Methods in Applied Sciences, vol. 18, no. 5, pp. 781-812, 2008.

[7] Z. Xiaofeng and R. J. McGough, "The Khokhlov-Zabolotskaya-Kuznetsov (KZK) equation with power law attenuation," in Proceedings of the IEEE International Ultrasonics Symposium (IUS '14), pp. 2225-2228, Chicago, Ill, USA, September 2014.

[8] T. Y. Cheung, Application of the Khokhlov-ZabolotskayaKuznetsov equation to modeling high-intensity focused ultrasound beams [M.S. thesis], Boston University, Boston, Mass, USA, 2008.

[9] W. I. Fushchich, W. M. Shtelen, and N. I. Serov, Symmetry Analysis and Exact Solutions of Equations of Nonlinear Mathematical Physics, vol. 246 of Mathematics and its Applications, Kluwer Academic, Dordrecht, The Netherlands, 1993.
[10] N. Euler and W.-H. Steeb, Continuous Symmetries, Lie Algebras and Differential Equations, Bibliographisches Institut, Mannheim, Germany, 1992.

[11] J. Biazar and F. Azimi, "He's homotopy perturbation method for solving Helmholtz equation," International Journal of Contemporary Mathematical Sciences, vol. 3, no. 13-16, pp. 739-744, 2008.

[12] R. Courant and D. Hilbert, Methods of Mathematical Physics, Vol 2: Partial Differential Equations, Interscience Publishers, New York, NY, USA, 1962.

[13] L. Debnath, Nonlinear Partial Differential Equations for Scientists and Engineers, Birkhäuser, Boston, Mass, USA, 1997.

[14] L. C. Evans, Partial Differential Equations, Graduate Studies in Mathematics, vol. 19, American Mathematical Society, 1999.

[15] F. John, Partial Differential Equations, Springer, New York, NY, USA, 1982.

[16] M. Khan, M. A. Gondal, and S. Kumar, "A novel homotopy perturbation algorithm for linear and nonlinear system of partial differential equations," World Applied Sciences Journal, vol. 12, no. 12, pp. 2352-2357, 2011.

[17] J.-H. He, "Variational principles for some nonlinear partial differential equations with variable coefficients," Chaos, Solitons \& Fractals, vol. 19, no. 4, pp. 847-851, 2004.

[18] G. Adomian and R. Rach, "Noise terms in decomposition solution series," Computers \& Mathematics with Applications, vol. 24, no. 11, pp. 61-64, 1992.

[19] G. Adomian, "A review of the decomposition method in applied mathematics," Journal of Mathematical Analysis and Applications, vol. 135, no. 2, pp. 501-544, 1988.

[20] G. Adomian and R. Rach, "Modified decomposition solution of linear and nonlinear boundary-value problems," Nonlinear Analysis: Theory, Methods \& Applications, vol. 23, no. 5, pp. 615619, 1994.

[21] J. Biazar and H. Ebrahimi, "An approximation to the solution of hyperbolic equations by Adomian decomposition method and comparison with characteristic method," Applied Mathematics and Computation, vol. 163, no. 2, pp. 633-638, 2005.

[22] H. Gu and Z.-B. Li, "A modified Adomian method for system of nonlinear differential equations," Applied Mathematics and Computation, vol. 187, no. 2, pp. 748-755, 2007.

[23] R. Jebari, I. Ghanmi, and A. Boukricha, "Adomian decomposition method for solving nonlinear heat equation with exponential nonlinearity,' International Journal of Mathematical Analysis, vol. 7, no. 15, pp. 725-734, 2013. 


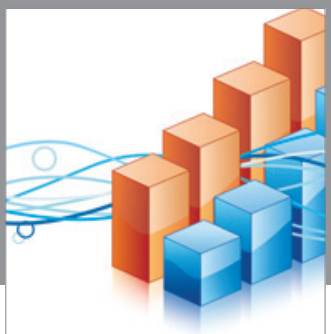

Advances in

Operations Research

vatem alat4

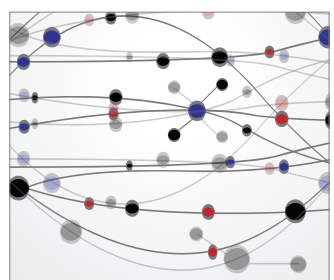

\section{The Scientific} World Journal
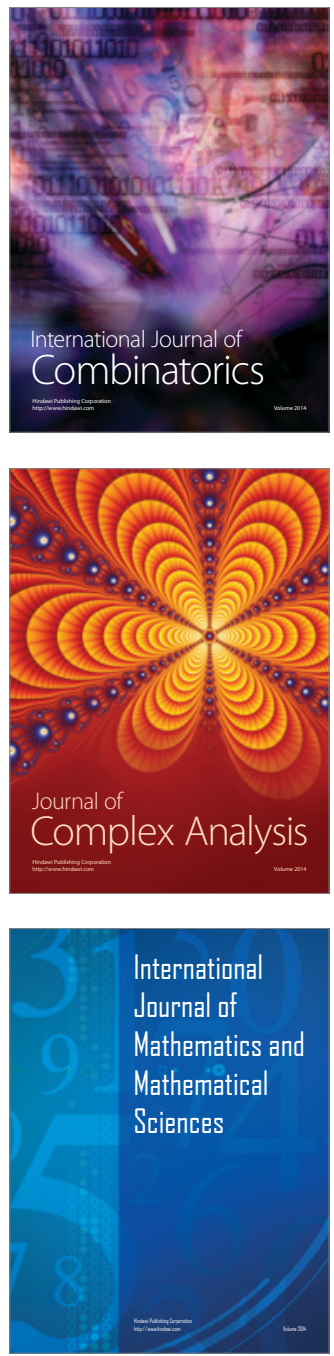
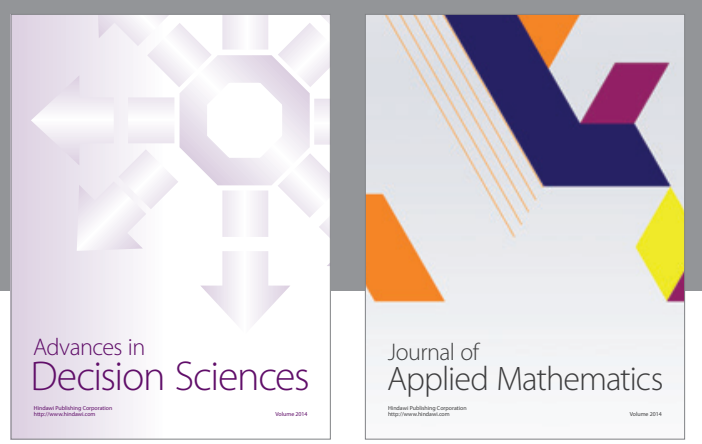

Algebra

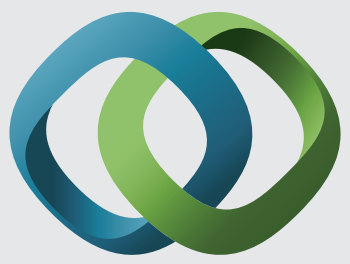

\section{Hindawi}

Submit your manuscripts at

http://www.hindawi.com
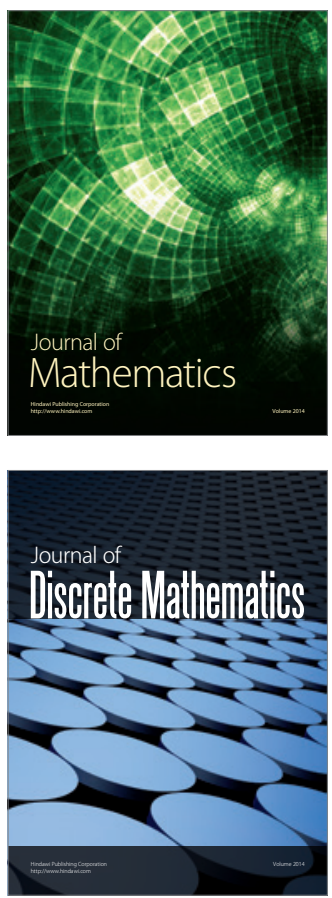

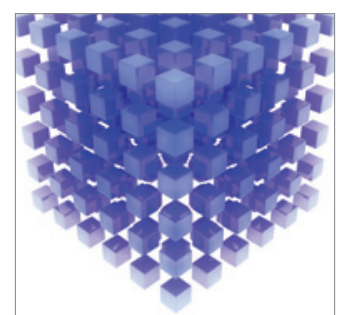

Mathematical Problems in Engineering
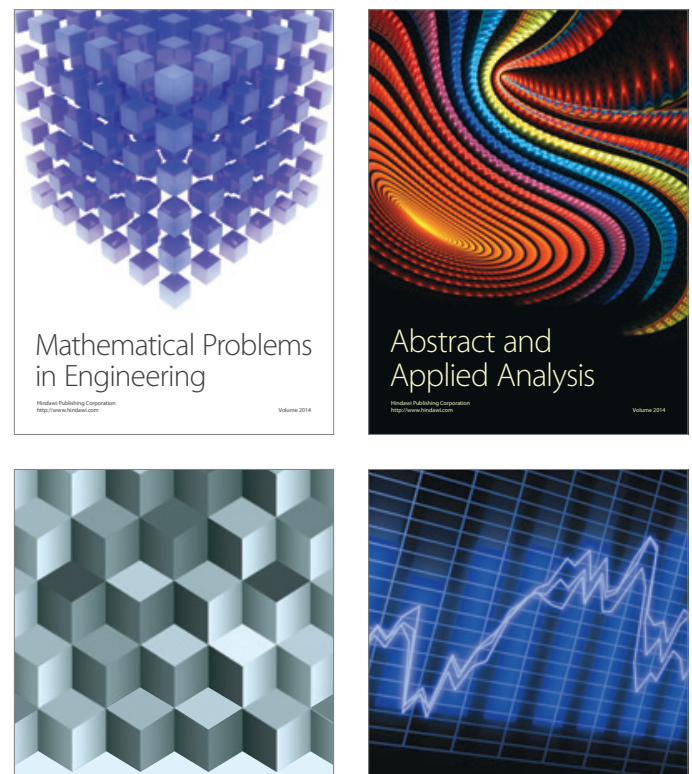

Journal of

Function Spaces

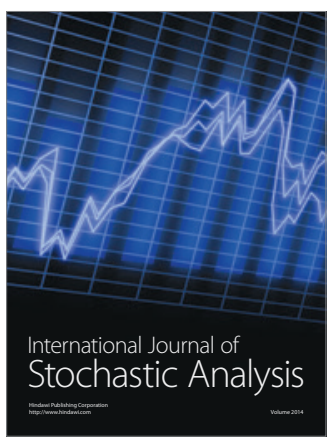

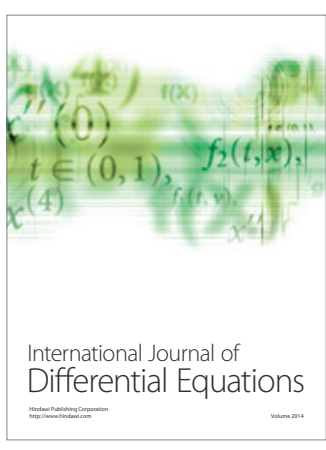
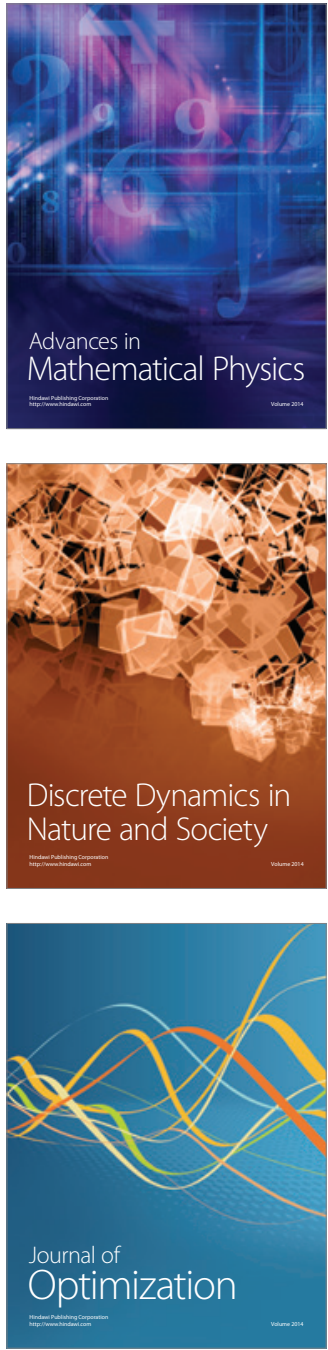